\title{
Wichtiger Entscheid für die Komplementärmedizin: Vier Therapierichtungen werden in die Grundversicherung aufgenommen
}

\section{Medienmitteilung des Eidgenössischen \\ Departements des Innern (2. Mai 2014)}

Die Leistungen der anthroposophischen Medizin, der traditionellen chinesischen Medizin (TCM), der Homöopathie und der Phytotherapie sollen grundsätzlich von der obligatorischen Krankenpflegeversicherung übernommen werden. Dabei sollen umstrittene Leistungen gezielt daraufhin überprüft werden, ob sie wirksam, zweckmässig und wirtschaftlich sind. Mit diesem Vorgehen möchte das Eidgenössische Departement des Innern (EDI) einem Verfassungsauftrag nachkommen.

Im Mai 2009 haben Volk und Stände den neuen Verfassungsartikel zur Besserstellung der Komplementärmedizin deutlich angenommen. Seit 2012 übernimmt die obligatorische Krankenpflegeversicherung (OKP) provisorisch bis 2017 ärztliche Leistungen der anthroposophischen Medizin, der TCM, der ärztlichen Homöopathie sowie der Phytotherapie.

Die Vergütung ist provisorisch und befristet, weil der Nachweis aussteht, dass die Leistungen der vier komplementärmedizinischen Fachrichtungen wirksam, zweckmässig und wirtschaftlich sind. Nach 2 Jahren zeichnet sich nun ab, dass dieser Nachweis für die Fachrichtungen als Ganzes nicht möglich sein wird.

Deshalb schlägt das EDI vor, diese Fachrichtungen den anderen von der OKP vergüteten medizinischen Fachrichtungen gleichzustellen. Damit gälte auch für sie das Vertrauensprinzip, und die Leistungen würden grundsätzlich von der OKP vergütet. Analog zu den anderen medizinischen Fachrichtungen sollen lediglich einzelne, umstrittene Leistungen daraus überprüft werden. Wie die Kriterien der Wirksamkeit, Zweckmässigkeit und Wirtschaftlichkeit (WZW) angewendet werden, muss dabei für die Komplementärmedizin noch präzisiert werden.

Das EDI und das Bundesamt für Gesundheit (BAG) haben die betroffenen Kreise über das geplante Vorgehen informiert und sie eingeladen, bei der Erarbeitung der Kriterien und Prozesse mitzuwirken.

Um dem Verfassungsauftrag auf diese Weise nachzukommen, müssen die Verordnung über die Krankenver- sicherung sowie die Verordnung des EDI über Leistungen in der OKP angepasst werden. Diese können der Bundesrat bzw. das EDI in eigener Kompetenz beschliessen.

\section{Stellungnahme des Dachverbands Komplementärmedizin}

Ärztliche Komplementärmedizin nicht mehr bestritten

Der Dakomed und die UNION begrüssen den Vorschlag des EDI zur definitiven Aufnahme der ärztlichen Komplementärmedizin in die OKP durch Gleichstellung mit anderen Fachrichtungen. Die definitive Vergütung durch die Grundversicherung ist überfällig. Der Dakomed und die UNION wünschen sich jetzt eine rasche Umsetzung der angekündigten Änderungen der KLV und KVV. Wir erwarten, dass alle betroffenen Kreise den Prozess positiv unterstützen.

Vor 5 Jahren, am 17. Mai 2009, haben die Stimmberechtigten mit einer Zweidrittelmehrheit dem Verfassungsartikel 118a zur Berücksichtigung der Komplementärmedizin im Schweizerischen Gesundheitswesen zugestimmt. Auch neueste Umfragen zeigen deutlich, dass die Komplementärmedizin von einer Mehrheit der Bevölkerung unterstützt und gewünscht wird. Eine der zentralen Forderungen ist die Vergütung der vier ärztlichen Fachrichtungen anthroposophisch erweiterte Medizin, klassische Homöopathie, Pflanzenheilkunde und TCM durch die Grundversicherung, wenn diese durch ärztliche Fachpersonen mit entsprechender Zusatzausbildung erbracht werden. (Die Leistungserbringung durch nichtärztliche TherapeutInnen wird dadurch nicht tangiert. Die Kostenübernahme dieser Leistungen erfolgt weiterhin über die entsprechenden Zusatzversicherungen.)

Die Würdigung aller in den letzten 13 Jahren erarbeiteten Evidenz erlaubt die Feststellung, dass der WZWNachweis für die ärztliche Komplementärmedizin heute vergleichbar ist mit der konventionellen Medizin. Die Qualitätssicherung ist durch die hohen Anforderungen an die fachärztlichen Aus- und Weiterbildungen gewährleistet: Die FMH-anerkannten Fähigkeitsausweise setzen

\section{KARGER}

Fax +497614520714
(C) 2014 S. Karger GmbH, Freiburg 
das vollgültige Staatsexamen, eine mindestens fünfjährige schulmedizinische Facharztausbildung sowie die Zusatzausbildung in mindestens einer der vier komplementärmedizinischen Fachrichtungen voraus. Damit steht der definitiven Aufnahme der ärztlichen Komplementärmedizin in die OKP nichts mehr im Wege.

\section{Stellungnahme von Dr. med. Hansueli Albonico, Präsident der UNION Schweizerischer komplementärmedizinischer Ärzteorganisationen}

Als UNIONs-Präsident begrüsse ich den Vorschlag des EDI zur Gleichstellung der ärztlichen Komplementärmedizin mit anderen Fachrichtungen und damit zu ihrer definitiven Aufnahme in der OKP.

Die Würdigung aller in den letzten 13 Jahren erarbeiteten Evidenz erlaubt die Feststellung, dass der WZWNachweis für die ärztliche Komplementärmedizin heute vergleichbar ist mit der konventionellen Medizin. Die Qualitätssicherung ist durch die hohen Anforderungen an die fachärztlichen Aus- und Weiterbildungen gewährleistet: Die FMH-anerkannten Fähigkeitsausweise setzen das vollgültige Staatsexamen, eine mindestens fünfjährige schulmedizinische Facharztausbildung sowie die $\mathrm{Zu}$ satzausbildung in mindestens einer der vier komplementärmedizinischen Fachrichtungen voraus.

Die Abstimmung vor 5 Jahren hat klargemacht: Wir brauchen nicht nur Hausärztinnen und Hausärzte, sondern auch komplementärmedizinisch kompetente Hausärztinnen und Hausärzte!

\section{Stellungnahme von Dr. med. Roger Eltbogen, Präsident SMGP}

Vertrauensprinzip - eine faire Art zur Beurteilung der Leistungen der Komplementärmedizin

Die Phytotherapie als gut dokumentierte Basis der Schulmedizin und damit die SMGP (Schweizerische Medizinische Gesellschaft für Phytotherapie) freut sich über die Aufnahme ihrer Leistungen in den Katalog der obligatorischen Krankenversicherung. Die Gleichstellung mit den anderen von der OKP vergüteten Fachrichtungen erachten wir als fair. Mit der Anwendung des Vertrauensprinzips erfüllt das EDI ein wichtiges Anliegen der Volksabstimmung vom Mai 2009.

Die Massnahme ist für die SMGP Motivation, die mit Unterstützung des Universitätsspitals Zürich und der Zürcher Hochschule für Angewandte Wissenschaften (ZHAW) etablierte Fort- und Weiterbildung weiter bekannt zu machen. Die Anerkennung führt hoffentlich zu einem vermehrten Interesse bei jungen Medizinerinnen und Medizinern, damit das patientenfreundliche hohe Potenzial der Phytotherapie auch in Zukunft der Medizin erhalten bleibt.

\section{Stellungnahme von Dr. Herbert Schwabl, Präsident SVKH/Geschäftsführer Padma AG}

\section{Wichtiger Paradigmenwechsel}

Die Anwendung des Vertrauensprinzips für die Aufnahme der ärztlichen Leistungen in die Grundversicherung kann man als einen wichtigen Paradigmenwechsel einstufen. Die Diskussion wird damit auf eine neue Ebene gehoben: Es geht nun nicht mehr darum, aus der Position des unbeliebten Antragstellers zu argumentieren. Nun können analog wie bei der Schulmedizin gezielt einzelne Leistungen geprüft werden, ohne jeweils die gesamte Fachrichtung unter Generalverdacht zu stellen. Der Dialog auf fachlicher Ebene ist die Garantie z.B. für ärztliche Leistung mit hoher Qualität und für die Ausbildung der jungen Ärztegeneration. Mit diesem Entscheid von Bundesrat Berset kann nun die ärztliche Komplementärmedizin diesen Weg fortsetzen.

Dieses Vertrauensprinzip ist auch für die Zulassung von natürlichen Heilmitteln eine Grundvoraussetzung. Das neue Heilmittelgesetz steht gerade in der parlamentarischen Beratung. Und auch hier geht es darum, die Qualität und Vielfalt der Heilmittel der Komplementärmedizin zu erhalten und zu stärken. 